\title{
Compatibility of MRI and Pathological Tumor Regression Grading in Patients with Locally Advanced Rectal Cancer After Undergoing Neoadjuvant Chemoradiotherapy
}

\author{
(1) Alaettin ARSLAN, ${ }^{1}$ (1) Gamze TÜRK, ${ }^{2}$ (I) Saliha KARAGÖZ EREN, ${ }^{3}$ (1) Ebru AKAY, ${ }^{4}$ (D) Nail ÖZHAN, ${ }^{5}$ \\ (1) İpek PInar ARAL ${ }^{6}$
}

'Department of Radiation Oncology, Kayseri City Hospital, Kayseri-Turkey

${ }^{2}$ Department of Radiology, Kayseri City Hospital, Kayseri-Turkey

${ }^{3}$ Department of Genaral Surgery, Kayseri City Hospital, Kayseri-Turkey

${ }^{4}$ Department of Pathology, Kayseri City Hospital, Kayseri-Turkey

${ }^{5}$ Department of Medical Oncology, Kayseri City Hospital, Kayseri-Turkey

${ }^{6}$ Department of Radiation Oncology, Nevsehir State Hospital, Nevsehir-Turkey

\begin{abstract}
OBJECTIVE
The compatibility between magnetic resonance imaging tumor regression grade (mrTRG) and pathological tumor regression grade (pTRG) was examined in patients with locally advanced rectal cancer (LARC) who underwent neoadjuvant chemoradiotherapy (nCRT). The primary endpoint of the study was to evaluate the relationship between mrTRG and PTRG after nCRT. The secondary endpoint of the study was to evaluate the variables that affect mrTRG and pTRG.
\end{abstract}

\section{METHODS}

Forty-one patients with LARC treated with nCRT were analyzed. Magnetic resonance imaging (MRI) performed after nCRT was compared with MRI taken before nCRT. Changes in T and $\mathrm{N}$ stages and extramural vascular invasion positivity were investigated. TRG was divided into five groups in terms of pathological and MRI. The Dworak pTRG system was used for compatibility testing. MRI sensitivity and specificity were evaluated by comparing MRI-based response assessment with pathological assessment after nCRT.

\section{RESULTS}

Median patient age was 61 years (range, 26-79 years), and 18 (43.9\%) were female. The relationship between mrTRG and pTRG was evaluated with the Cohen kappa coefficient. Significant compatibility was observed between pTRG and mrTRG ( $\mathrm{p}=0.002$ ), but the compatibility was low (kappa compatibility, $0.319)$. The sensitivity of mrTRG was $90 \%(18 / 20)$; specificity was $14.3 \%(3 / 21)$; positive predictive value was $85.7 \%(18 / 21)$; and negative predictive value was $90 \%(18 / 20)$. While pTRG was negatively affected by advanced age ( $\mathrm{p}=0.037)$, mrTRG was adversely affected by advanced post-nCRT $\mathrm{N}$ stage $(\mathrm{p}=0.048)$.

\section{CONCLUSION}

As age increased, pTRG was negatively affected; as the post-nCRT N stage increased, mrTRG was negatively affected. There was compatibility between mrTRG and pTRG, as expected, but this compatibility was found to be low.

Keywords: MRI; neoadjuvant chemoradiotherapy; rectal cancer; tumor regression grade.

Copyright $\odot$ 2021, Turkish Society for Radiation Oncology

Dr. Alaettin ARSLAN

Kayseri Şehir Hastanesi,

Radyasyon Onkolojisi Anabilim Dalı,

Kayseri-Turkey

E-mail: alaettin.arslan@gmail.com 


\section{Introduction}

Magnetic resonance imaging (MRI) is the most commonly used diagnostic method for radiologically imaging rectal cancer. MRI has advantages in the evaluation of locally advanced stage rectal cancer (LARC). With rectal MRI, it is possible to determine many tumor lesion features, including size, morphology, and borders, which are useful in treatment planning.[1,2] MRI is used both before and after neoadjuvant chemoradiotherapy (nCRT). Significant progress has been made in controlling the local disease using nCRT in cases with LARC detected by MRI. At present, the most preferred imaging method for local staging of rectal cancer is rectal MRI.[3,4] Due to the increasing prominence of nCRT in recent years, the importance of imaging techniques has been studied, for both staging and accurately evaluating radiological response. Tumor volume reduction and fibrotic transformation are the two primary response markers that can be appreciated on morphological (T2 weighted) MRI and can help guide the treatment strategy after nCRT. However, research has indicated that morphological MRI is unable to differentiate between sterile fibrosis and fibrosis-containing tumor tissue, which is a significant clinical limitation. Researchers have concluded that it would be appropriate to add diffusion-weighted imaging to the MRI protocol, stating that diffusion-weighted MRI successfully distinguishes between tumor and fibrosis.[5]

Pathological tumor regression grade (pTRG) is a system used in the histological evaluation of tumor response to nCRT that was initially used in other cancers of the gastrointestinal system, bladder, head, and neck. [6-9] Overtime, studies were carried out on the use of pTRG in determining the prognosis of LARC. $[10,11]$ The most well-known pTRG systems are Mandard, Rödel, Dworak, and the American Joint Committee on Cancer (AJCC) scoring systems.[7,12-14] Recently, a pTRG-like MRI tumor regression grade (mrTRG) system was developed.[15,16] The basic principle of both the mrTRG and PTRG systems is related to tumor fibrosis rate following nCRT. Radiological interpretation requires the comparison of high-resolution oblique images with basal scans to determine the proportions of the tumor with fibrotic low signal intensity and residual medium signal intensity. There has been a lack of consensus in previous studies on the compatibility of mrTRG and pTRG in patients with LARC. In the study by Sclafani et al.,[16] agreement between mrTRG and pTRG was found to be poor, and mrTRG was seen as a complementary prognostic tool for surgical decision- making and pTRG. In contrast, Rengo et al.[17] found the compatibility between mrTRG and pTRG to be excellent. Ko et al.[18] recommended combining MRI and endoscopic biopsy to detect complete remission.

The histopathological definition of extramural vascular invasion (EMVI) is tumor cells invading vessels beyond the muscularis propria, which indicates a poor prognosis in patients with rectal cancer.[19] MRI can provide accurate guidance in detecting EMVI (mrEMVI).[20] Several studies have shown that mr-EMVI is one of the risk factors for distant metastasis in rectal cancer.[21,22] Particularly in patients with a baseline T3 stage, defining EMVI may be important in predicting patients' response to nCRT and long-term results.[23]

In this study, changes in $\mathrm{T}$ and $\mathrm{N}$ stages and the positivity of EMVI were investigated. In addition, the compatibility of mrTRG and pTRG was examined to investigate the reliability of MRI in the diagnosis and treatment of rectal cancer.

\section{Materials and Methods}

\section{Patient Selection}

A total of 72 patients with a diagnosis of rectal cancer who received nCRT in the radiation oncology clinic of our hospital between June 2014 and July 2019 were evaluated. Patient interview information, patient files, and electronic system data were used for data collection. The patients' demographic status, primary diagnosis, disease stage, tumor localization, distance to the anal sphincter, MRI examination, treatment method, treatment response, and final status were noted.

Patients older than 18 years of age with pathological evidence of rectum adenocarcinoma, Eastern Cooperative Oncology Group 1-2, locally advanced stage according to AJCC eighth edition, complete imaging and treatment information, and nCRT treatment were included in the study. Ten patients with missing files, follow-up information, or MRI imaging were excluded from the study. In addition, 21 patients who were prescribed nCRT but were not operated on for any reason were excluded from the study. The remaining 41 patients were included in the study.

\section{Imaging and Treatment Details}

Before treatment, all patients were evaluated by the multidisciplinary treatment council including general surgery, medical oncology, and radiation oncology. Digital examination of the rectum, colonoscopy, multislice triphasic thorax, abdominal, and pelvic com- 
puted tomography, endorectal ultrasonography (USG), and pelvic MRI was ordered in the initial evaluation. Pelvic MRIs were obtained with pelvic phased-array coils and 1.5 T MRI. Pelvic MRI consisted of sagittal fast spin-echo T2-weighted images, coronal short-au inversion recovery, axial fast spin-echo T2-weighted images, axial T2-weighted images with fat saturation, axial T1-weighted images, axial T1-weighted images with gadolinium, and sagittal T1-weighted images with gadolinium. Table 1 shows the parameters for pelvic MRI sequences.

First, sagittal T2-weighted images were obtained, and after a segment containing tumor tissue was detected, this segment was scanned transversely. EMVI was evaluated with axial T2-weighted images and axial T1-weighted images with gadolinium. On MRI, EMVI is characterized by focal enlargement of the vessel, wall irregularity, and loss of flow void. Diffusion-weighted MRI was not obtained. Fibrosis was evaluated with T2weighted and post-contrast T1-weighted images.

The nCRT treatment was performed with Varian DHX and IX devices at Kayseri Training and Research Hospital and Kayseri City Hospital. A total dose of 45 Gy with a fraction dose of 180 cGy was administered 5 days a week for 5 weeks. For the radiotherapy (RT) technique, 3-field 3-dimensional conformal RT (3DCRT), double-arc volumetric arc therapy, and 7-field intensity-modulated RT were used. No additional dose was administered to the mesorectum due to the feedback regarding the difficulties experienced in surgical operations due to RT. RT was applied to the rectum and mesorectum as well as to the internal iliac, obturator, and presacral lymph nodes. Capecitabine was administered as a chemotherapy (ChT) agent at a dose of 825 $\mathrm{mg} / \mathrm{m}^{2}$ twice a day, 5 days a week concurrent with RT. Patients underwent surgical operation an average of 68 days (range, 33-385 days) after nCRT. Total mesorec- tal excision (TME) was performed on all patients. Capecitabine plus oxaliplatin, oxaliplatin plus leucovorin plus 5-FU, and capecitabine were administered to the patients as adjuvant ChT. The patients were evaluated before treatment, 1 and 2 months after the end of nCRT, and every 3 months after the operation. During the evaluation, blood tests were performed and the tumor markers carcinoembryonic antigen and carbohydrate antigen 19-9 were evaluated. Abdominal USG and pelvic MRIs were performed for all patients.

\section{Primary and Secondary Endpoints}

The primary endpoint of the study was to evaluate the relationship between mrTRG and pTRG after treatment. In accordance with the literature, tumor response grade was divided into five groups for both pathological and MRI methods.[13,15,16] The grading paradigms for the Dworak pTRG system and the mrTRG system are presented in Table 2. Patel et al.[15] demonstrated the prognostic significance of reassessing rectal cancers using high-resolution T2-weighted MRI after completing nCRT.[15] In our study, based on the previous studies, the MRI response was divided into two groups: Good response (GR; mrTRG1-2) and poor response (PR; mrTRG3-5).

Similarly, the pathological response was dichotomized as GR (pTRG3-4) and PR (pTRG0-2). $[24,25]$ MRI sensitivity and specificity were evaluated by comparing the MRI-based response assessment with pathological results after neoadjuvant therapy. The secondary endpoint of the study was to evaluate the variables that affect mrTRG and pTRG.

\section{Statistical Analysis}

Descriptive statistics for continuous (quantitative) variables were expressed as mean, standard deviation, minimum-maximum, and median values, while categori-

Table 1 Technical information on pre-nCRT and post-nCRT MRIs

T2 sagittal

T2 transverse

STIR coronal

T2 transverse

T1 transverse

$\mathrm{T} 1$ transverse $+\mathrm{C}$

T1 sagittal $+C$

\section{TE}

5100
5100
4090
7350
597
836
597

TR

TI

$90-150$
$90-150$
49
88
21
21
21

FA

180
180
160
180
180
180
180

FOV

$\begin{array}{ll}270 \times 300 & 4 / 1 \\ 334 \times 370 & 4 / 1 \\ 382 \times 348 & 4 / 1 \\ 334 \times 370 & 4 / 1 \\ 334 \times 370 & 4 / 1 \\ 334 \times 370 & 4 / 0 \\ 350 \times 350 & 4 / 0\end{array}$

STIR: Short-tau inversion recovery; $+C$ with contrast scan, $n C R T$ : Neoadjuvant chemoradiotherapy, MRI: Magnetic resonance imaging; TE: Time to echo; TR: Repetition time; TI: Inversion time; FA: Flip angle; FOV: Field of view 
Table 2 The classification system for mrTRG and pTRG

mrTRG

Tumor appears unchanged

Tumor predominance with minimal low signal fibrosis

Moderate regression (low signal intensity fibrosis predominates, but there are obvious areas of intermediate

There is minimal residual intermediate tumor signal.

Complete regression (absence of tumor signal and barely visible treatment-related scar)

PTRG/Dworak

\section{Description}

No regression

Dominant tumor mass with obvious fibrosis and/or vasculopathy

Dominantly fibrotic changes with few tumor cells or groups (easy to find)

Very few (difficult to find microscopically) tumor cells in fibrotic tissue with or without mucous substance

No tumor cells, only fibrotic mass (total regression or response)

mrTRG: Magnetic resonance imaging tumor regression grade; pTRG: Pathological tumor regression grade

cal variables were expressed as number (n) and ratio (\%). Cohen's Kappa analysis was used for mrTRG and pTRG compatibility $(\kappa<0.20$ : poor agreement; $\kappa=0.21$ 0.40 : fair agreement; $\kappa=0.41-0.60$ : moderate agreement; $\kappa=0.61-0.80$ : good agreement; and $\kappa=0.81-1.00$ : very good agreement). Non-parametric tests were used in the study, the Mann-Whitney U-test for statistical analysis of two independent groups and the KruskalWallis test for analysis of three or more independent groups. Significance was evaluated with post hoc analysis after Bonferroni correction. Chi-square and Fisher's exact tests were used to calculate the categorical demographic characteristics of the patients. Analyses were performed with IBM SPSS Package Program version 24.0 (IBM Corporation, Armonk, NY, USA). Statistical significance was determined as $\mathrm{p}<0.05$.

\section{Results}

The results of 41 patients diagnosed with LARC who received curative treatment in our clinic were evaluated. The patients' median age was 61 years (range, 26-79 years), and 18 (43.9\%) were female. All patients had prenCRT and pre-operative comparative functional MRI results. Only 2 patients $(4.9 \%)$ had high rectal localization, while $19(46.3 \%)$ patients had midrectal localization and $20(48.8 \%)$ patients had low rectal localization. Radiologically, EMVI was positive in $16(39 \%)$ patients on MRI before nCRT, and pathological lymph node $(\mathrm{pLN})$ was positive in $34(82.9 \%)$ patients. A majority of patients underwent 3D-CRT $(56.1 \%, n=23)$. Patient characteristics and treatment details are summarized in Table 3.

\section{Radiological Effect of nCRT on EMVI}

While 16 patients (39\%) had EMVI positivity on prenCRT MRI, the number of EMVI-positive patients decreased to $7(17.1 \%)$ on post-nCRT MRI. Although the number of EMVI-positive patients decreased after nCRT, the difference was not statistically significant ( $>0.05)$.

\section{Evaluation of mrTRG and Dworak pTRG Rela- tionship}

The relationship between mrTRG and Dworak pTRG was assessed using the Cohen kappa fit analysis test. A low level but significant $(\kappa=0.319, \mathrm{p}=0.002)$ compatibility was observed between pTRG and mrTRG (Table 4).

GR was observed in 21 (51.2\%) patients and PR was observed in 20 (48.8\%) patients according to basal images in the post-nCRT MRI. GR was observed in 20 (48.8\%) patients and PR was observed in 21 (51.2\%) patients based on the pathological evaluation.

When the accuracy of mrTRG in treatment response evaluation was compared with pTRG, mrTRG was determined to have a sensitivity of $90 \%(18 / 20)$, specificity of $14.3 \%(3 / 21)$, positive predictive value of $85.7 \%$ (18/21), and negative predictive value of $90 \%$ $(18 / 20)$ (Table 5). 
Table 3 Patient characteristics and treatment details

\begin{tabular}{|c|c|c|c|c|c|}
\hline Parameters & $\mathbf{n}$ & $\%$ & Parameters & $\mathbf{n}$ & $\%$ \\
\hline Age & & & Stage T (pre-nCRT MRI) & & \\
\hline Median & 61 & (range 26-79) & T3a & 9 & (22) \\
\hline \multicolumn{6}{|l|}{ Gender } \\
\hline Female & 18 & $(43.9)$ & $\mathrm{T} 3 \mathrm{~b}$ & 13 & (31.7) \\
\hline Male & 23 & $(56.1)$ & T3c & 11 & $(26.8)$ \\
\hline \multicolumn{6}{|l|}{ Localization } \\
\hline Low & 20 & $(48.8)$ & T3d & 2 & (4.9) \\
\hline Middle & 19 & $(46.3)$ & $\mathrm{T} 4 \mathrm{a}$ & 4 & (9.8) \\
\hline High & 2 & $(4.9)$ & $\mathrm{T} 4 \mathrm{~b}$ & 2 & (4.9) \\
\hline EMVI (pre-nCRT MRI) & & & Stage T (post-nCRT MRI) & & \\
\hline Positive & 16 & (39) & $\mathrm{T} 2$ & 9 & (22) \\
\hline Negative & 25 & (61) & T3a & 17 & $(41.5)$ \\
\hline \multicolumn{6}{|l|}{ EMVI (post-nCRT MRI) } \\
\hline Positive & 7 & $(17.1)$ & T3b & 8 & (19.5) \\
\hline Negative & 34 & (82.9) & T3c & 2 & $(4.9)$ \\
\hline \multicolumn{6}{|l|}{ pLN (pre-nCRT MRI) } \\
\hline Positive & 34 & (82.9) & T4a & 3 & (7.3) \\
\hline Negative & 7 & $(17.1)$ & $\mathrm{T} 4 \mathrm{~b}$ & 2 & (4.9) \\
\hline pLN (post-nCRT MRI) & & & Stage N (pre-nCRT MRI) & & \\
\hline Positive & 13 & (31.7) & No & 6 & (14.6) \\
\hline Negative & 28 & $(68.3)$ & N1a & 7 & $(17.1)$ \\
\hline \multicolumn{6}{|l|}{ RT technique } \\
\hline 3D-CRT & 23 & $(56.1)$ & $\mathrm{N} 1 \mathrm{~b}$ & 14 & $(34.1)$ \\
\hline ARC & 6 & $(14.6)$ & N1c & 4 & (9.8) \\
\hline IMRT & 12 & $(29.3)$ & $\mathrm{N} 2 \mathrm{a}$ & 6 & $(14.6)$ \\
\hline \multicolumn{6}{|l|}{ RT dose } \\
\hline $45 \mathrm{~Gy} / 25 \mathrm{frx}$ & 41 & $(100)$ & $\mathrm{N} 2 \mathrm{~b}$ & 4 & (9.8) \\
\hline cCT agent & & & Stage N (post-nCRT MRI) & & \\
\hline Capecitabine & 41 & (100) & No & 29 & (70.7) \\
\hline \multicolumn{6}{|l|}{ Adjuvant CT } \\
\hline Yes & 41 & (100) & N1a & 7 & $(17.1)$ \\
\hline No & 0 & (0) & $\mathrm{N} 1 \mathrm{~b}$ & 3 & $(7.3)$ \\
\hline \multicolumn{6}{|l|}{ Adjuvant $\mathrm{ChT}$ regimens } \\
\hline XELOX & 8 & (19.6) & N1c & 2 & (4.9) \\
\hline $\mathrm{XELOX}_{2}$ & 13 & $(31.7)$ & & & \\
\hline FOLFOX6 & 11 & $(26.8)$ & & & \\
\hline Capecitabine & 9 & $(21.9)$ & & & \\
\hline \multicolumn{6}{|l|}{ Between nCRT-MRI } \\
\hline Day & 39 & (range 12-83) & & & \\
\hline \multicolumn{6}{|l|}{ Between MRI-OP } \\
\hline Day & 29 & (range 5-352) & & & \\
\hline
\end{tabular}

EMVI: Extramural vascular invasion; CRT: Chemoradiotherapy; RT: Radiotherapy; OP: Operation; pLN: Pathological lymph node; 3D-CRT: Three-dimensional conformal RT; ARC: Volumetric arc therapy; IMRT: Intensity-modulated RT; XELOX: Capecitabine plus oxaliplatin; FOLFOX6: Oxaliplatin plus leucovorin plus 5-FU; cChT: Concurrent CT; nCRT: Neoadjuvant chemoradiotherapy; MRI: Magnetic resonance imaging; Gy: Gray; CT; Chemotherapy; XELOX Capecitabine 1500 mg/m²+Oxaliplatine 130 mg/ $\mathrm{m}^{2}$ (6 cycles every 21 days), XELOX Capecitabine $2000 \mathrm{mg} / \mathrm{m}^{2}+$ Oxaliplatine $130 \mathrm{mg} / \mathrm{m}^{2}$ (6 cycles every 21 days), FOLFOX6 Oxaliplatin $100 \mathrm{mg} / \mathrm{m}^{2}+$ Leucovorin $400 \mathrm{mg} /$ $\mathrm{m}^{2}+5$-FU $400 \mathrm{mg} / \mathrm{m}^{2}$ IV bolus, followed by $2400 \mathrm{mg} / \mathrm{m}^{2} 46 \mathrm{~h} 5$-FU infusion ( 12 cycles every 14 days), Capecitabine $2000 \mathrm{mg} / \mathrm{m}^{2}$ ( 6 cycles every 21 days)

\section{Analysis of Variables and pTRG}

No significant relationships were found between pTRG and gender, tumor localization, pre-nCRT EMVI, post-nCRT EMVI, pre-nCRT pLN, post-nCRT pLN,
pre-nCRT T stage, post-nCRT T stage, pre-nCRT N stage, post-nCRT $\mathrm{N}$ stage, pre-nCRT tumor length, post-nCRT tumor length, pre-nCRT tumor diameter, post-nCRT tumor diameter, RT technique, pathologi- 
Table 4 Detailed analysis of the relationship between mrTRG and pTRG

\begin{tabular}{|c|c|c|c|c|c|c|c|c|c|}
\hline & \multirow[b]{2}{*}{ TRG } & \multicolumn{5}{|c|}{ mrTRG } & \multirow[t]{2}{*}{ Total } & \multirow[t]{2}{*}{ Kappa } & \multirow[t]{2}{*}{$\mathbf{p}$} \\
\hline & & TRG1 & TRG2 & TRG3 & TRG4 & TRG5 & & & \\
\hline \multirow{5}{*}{ pTRG/Dworak } & TRG0 & 0 & 0 & 0 & 0 & 1 & 1 & 0.319 & 0.002 \\
\hline & TRG1 & 0 & 0 & 8 & 1 & 0 & 9 & & \\
\hline & TRG2 & 0 & 3 & 8 & 0 & 0 & 11 & & \\
\hline & TRG3 & 5 & 12 & 2 & 0 & 0 & 19 & & \\
\hline & TRG4 & 1 & 0 & 0 & 0 & 0 & 1 & & \\
\hline Total & & 6 & 15 & 18 & 1 & 1 & 41 & & \\
\hline
\end{tabular}

Cohen's Kappa analysis: mrTRG and pTRG compatibility ( $\mathrm{k}<0.20$ : poor agreement; $\mathrm{k}=0.21-0.40$ : Fair agreement; $\mathrm{K}=0.41-0.60$ : Moderate agreement; $\mathrm{K}=0.61-0.80$ : good agreement; and $\mathrm{k}=0.81-1.00$ : Very good agreement). Statistical significance $p<0.05$. mrTRG: Magnetic resonance imaging tumor regression grade; TRG: Tumor regression grade; pTRG: Pathological tumor regression grade

Table 5 MRI pathological response relationship after neoadjuvant therapy

\begin{tabular}{|c|c|c|c|c|}
\hline & \multicolumn{2}{|c|}{ pTRG/Dworak } & \multirow[t]{2}{*}{ Total } & \multirow[t]{2}{*}{$\mathbf{p}$} \\
\hline & GR (pTRG3-4) & PR (pTRG0-2) & & \\
\hline \multicolumn{5}{|l|}{ mrTRG } \\
\hline \multicolumn{5}{|l|}{ GR (mrTRG1-2) } \\
\hline $\mathrm{N}$ & 18 & 3 & 21 & $<0.001$ \\
\hline Row percentage, \% & 85.7 & 14.3 & 100.00 & \\
\hline Column percentage, $\%$ & 90.0 & 4.3 & 51.2 & \\
\hline \multicolumn{5}{|l|}{ PR (mrTRG3-5) } \\
\hline $\mathrm{N}$ & 2 & 18 & 20 & \\
\hline Row percentage, \% & 10 & 90 & 100.00 & \\
\hline Column percentage, $\%$ & 10 & 85.7 & 16.70 & \\
\hline \multicolumn{5}{|l|}{ Total } \\
\hline $\mathrm{N}$ & 20 & 21 & 41 & \\
\hline Row percentage, $\%$ & 48.8 & 51.2 & 100.00 & \\
\hline Column percentage, $\%$ & 100.00 & 100.00 & 100.00 & \\
\hline
\end{tabular}

The sensitivity of mrTRG was $90 \%$ (18/20); specificity was $14.3 \%$ (3/21); positive predictive value was $85.7 \%$ (18/21); and negative predictive value was $90 \%$ $(18 / 20)$. Statistical significance $p<0.05$. Chi-square test (Fisher's exact test). MRI: Magnetic resonance imaging; mrTRG: Magnetic resonance imaging tumor regression grade; pTRG: Pathological tumor regression grade; GR: Good response; PR: Poor response

cal subtype, and time between the end of RT and the operation ( $\mathrm{p}>0.05)$.

The median age of patients with GR based on pTRG was 57 years (range, 26-73 years), and the median age of patients with PR based on PTRG was 64 years (range, 41-79 years). As age increased, a significantly higher rate of $\mathrm{PR}$ was observed ( $\mathrm{p}=0.037$ ) (Fig. 1).

\section{Analysis of Variables and mrTRG}

No significant relationships were found between mrTRG and age, gender, tumor location, pre-nCRT EMVI, post-nCRT EMVI, pre-nCRT pLN, post-nCRT pLN, pre-nCRT $\mathrm{T}$ stage, post-nCRT $\mathrm{T}$ stage, prenCRT $\mathrm{N}$ stage, pre-nCRT tumor length, post-nCRT tumor length, pre-nCRT tumor diameter, post-nCRT

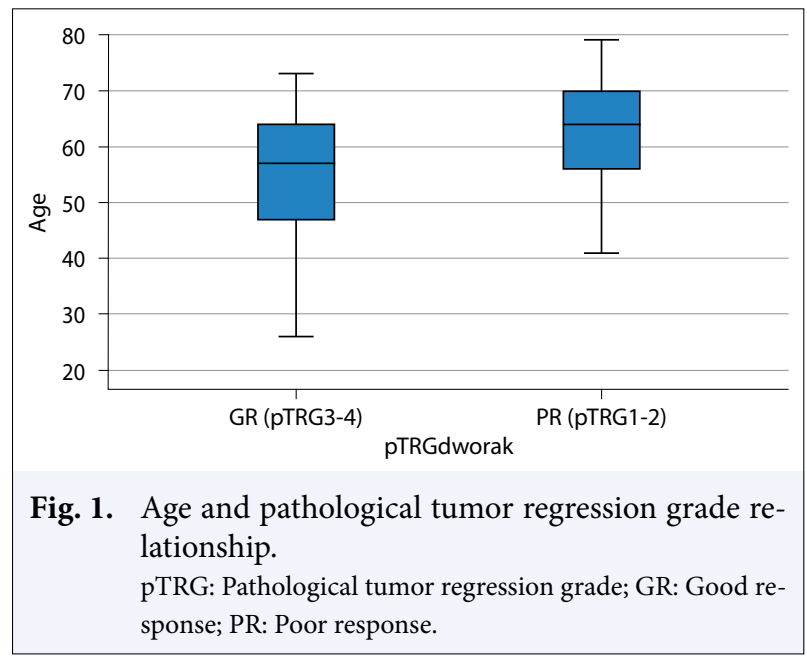


Table 6 mrTRG and post-nCRT nodal stage analysis

\begin{tabular}{lccccc}
\hline Post-nCRT N stage & GR (mrTRG1-2) (\%) & PR (mrTRG3-5) (\%) & Total & $\mathbf{X}^{\mathbf{2}}$ & $\mathbf{p}$ \\
\hline N0 & $17(58.6)$ & $12(41.4)$ & 29 & 5984 & $\mathbf{0 . 0 4 8}$ \\
N1a & $4(57.1)$ & $3(42.9)$ & 7 & & \\
N1b & $0(0)$ & $3(100)$ & 3 & & \\
N1c & $0(0)$ & $2(100)$ & 2 & & \\
\hline
\end{tabular}

Statistical significance $\mathrm{p}<0.05$. mrTRG: Magnetic resonance imaging tumor regression grade; $\mathrm{nCRT}$ : Neoadjuvant chemoradiotherapy; GR: Good response; PR: Poor response

tumor diameter, RT technique, pathological subtype, and time between the end of RT and MRI ( $p>0.05$ ).

The relationship between response based on $\mathrm{mrTRG}$ and post-nCRT $\mathrm{N}$ stage was significant ( $\mathrm{p}=0.048$ ); as the $\mathrm{N}$ stage progressed, the ratio of patients with $\mathrm{PR}$ increased significantly (Table 6).

\section{Discussion}

The stage of the disease and surgical margins largely determines the prognosis of patients with rectal cancer.[24] In addition, venous invasion, perineural invasion, and tumor grade impact prognosis.[26] EMVI is defined histologically as the involvement of veins other than the muscularis propria.[25] The presence of EMVI is an independent predictor of local or distant recurrence, nodal disease, and lower overall survival. Comparing pre-treatment and post-treatment scans, it has been shown that MRI has a high sensitivity even when advanced histopathological techniques are used to define EMVI.[27] MRI is currently the standard method for post-nCRT local staging of rectal cancer and has demonstrated good accuracy in identifying EMVI.[28] In our study, the prognostic value of EMVI was not examined, but the effect of nCRT on EMVI was examined to emphasize the importance of EMVI presence. The number of EMVI-positive patients decreased when prenCRT and post-nCRT MRIs were compared. The number of patients with positive EMVI on MRI decreased from 16 (39\%) pre-nCRT to 7 (17.1\%) post-nCRT.

In recent years, compatibility between mrTRG and pTRG has been the subject of multiple studies. Particularly in rectal cancer, the watch-and-wait approach has increased the importance of mrTRG. However, mrTRG is not a routinely offered service in every center. In addition, mrTRG findings have not been as consistent as pTRG findings, and studies have produced conflicting results regarding compatibility.

In the study by Siddiqui et al.,[29] mrTRG evaluation of the images of 12 patients by 35 radiologists was compared. Eight radiologists showed very good to near-perfect agreement $(\kappa>0.8), 6$ showed good agreement $(0.8 \geq \kappa>0.6)$, and 12 showed moderate agreement $(0.6 \geq \kappa>0.4)$. The radiologists identified good responders in $65.9 \%$ of cases and poor responders in $90 \%$ of cases. Although there was a trend toward good agreement among the radiologists, it is clear that there were still differing assessments. Since our study was retrospective, mrTRG evaluation by a single radiologist was specifically requested. Evaluation of all 41 cases by the same radiologist was deemed appropriate for the stability of the study. The conclusion of the previous study emphasized that radiologists can be taught mrTRG measurement and that good agreement can be achieved between radiologists.

Similar to the differences seen between studies in the evaluation of mrTRG, different results have been obtained regarding the agreement between mrTRG and pTRG. In research investigating the correlation between mrTRG and pTRG, 191 patients were evaluated and mrTRG and PTRG findings were compared in two Phase-II studies (EXPERT and EXPERT-C).[16] Patients were given 4 cycles of induction capecitabine and oxaliplatin (CAPOX) ChT first, and then, capecitabinebased concurrent nCRT (54 Gy in EXPERT; 50.4 Gy in EXPERT-C) was administered. TME was administered 4-6 weeks after the end of nCRT and 4 cycles of adjuvant ChT (capecitabine in EXPERT; CAPOX in EXPERT-C) were given. High-resolution MRI was taken at the end of induction ChT and 4 weeks after concurrent nCRT. The compatibility between mrTRG and pTRG was evaluated by the weighted kappa test. Fair agreement was found between mrTRG and pTRG when the regression was classified according to standard 5-tier systems $(\kappa=0.24)$ or modified 3-tier systems $(\kappa=0.25)$. The sensitivity and specificity of mrTRG1-2 (complete/good radiological regression) for the prediction of pathological complete response (pCR) were 74.4\% (95\% confidence interval [CI]: 58.8-86.5) and $62.8 \%$ (95\% CI: 54.5-70.6), respectively. The study 
concluded that the compatibility between mrTRG and pTRG was low and that mrTRG could not be used as a proxy for pTRG. It was emphasized that mrTRG can provide complementary prognostic information to pTRG for improved post-operative risk stratification and identify complete pathological responders for adopting non-operative management strategies, with the need for further studies on this topic highlighted. In another study, the MRIs of 65 patients were evaluated to quantitatively assess the percentage of fibrosis developing in LARC after nCRT.[17] The patients were followed for 30 months. Fibrosis percentage was measured on T2-weighted images, and mrTRG classes were determined and compared with histopathological pTRG. The compatibility between pTRG and mrTRG was determined to be perfect $(\kappa=0.923)$. The automated measurement of fibrosis detected by MRI has thus been recorded as feasible and reproducible. Significant agreement was found in our study between mrTRG and pTRG $(\mathrm{p}=0.002)$, though the agreement was low $(\kappa=0.319)$. When compared to Sclafani et al.,[16] the number of patients in our study was markedly less. In addition, in our study, RT dose was 45 Gy, capecitabine was used as the standard ChT, and only standard 5-tier systems were used. Despite differences in methodology, our study results were similar to those of Sclafani et al.[16] in terms of the low agreement measured between mrTRG and pTRG. Our sample size of 41 was more similar to the sample size of 65 in Rengo et al.,[17] though very different results were obtained in the compatibility of mrTRG and pTRG grades. Rengo et al.[17] measured the percentage of fibrosis on T2weighted images using the automated $\mathrm{k}$-means clustering algorithm. In our study, the percentage of fibrosis was measured visually on T2-weighted MRIs by the radiologist. The reason for the differences between the two studies may be due to the differences in methodology for measuring the percentage of fibrosis.

A different approach has been employed by Ko et al.[18] In their study, 102 patients who underwent an endoscopy and MRI 2-4 weeks after nCRT were examined retrospectively.[18] A confidence interval (1-4) was established for endoscopy and MRI evaluations. Accuracy, sensitivity, and specificity were analyzed based on the findings of endoscopy, MRI, and a combination method. Patients simultaneously received a total of 50.4 Gy RT from 3 to 4 fields ( 45 Gy pelvic and $5.4 \mathrm{~Gy}$ boost) and capecitabine, and pCR was achieved in $17(16.7 \%)$ of 102 patients. The accuracy, sensitivity, and specificity of pCR prediction from biopsy and endoscopy were $85.3 \%, 52.9 \%$, and $91.8 \%$, respectively, while from MRI, they were $91.2 \%, 70.6 \%$, and $95.3 \%$, respectively. With combined endoscopy and MRI, accuracy, sensitivity, and specificity were $89.2 \%, 52.9 \%$, and $96.5 \%$, respectively. No significant differences were found in the sensitivity and specificity of each method. The pCR prediction rate using the combination method was $92.6 \%$ after the final probability test. The study showed that the combination of endoscopic biopsy and MRI could positively predict pCR in patients with rectal cancer after nCRT.

No consensus has thus far been reached on the utility of mrTRG. In fact, mrTRG is not routinely evaluated in all centers. Therefore, more training and further studies are needed. In our study, we retrospectively investigated the compatibility of mrTRG and pTRG with a small patient group. Although compatibility was found to be low, these results are not decisive, as differing results have been obtained in other studies. At this stage, mrTRG cannot replace pTRG, but has prognostic value in the watch-and-wait patient group whose operations are delayed.

\section{Limitations of the Study}

The limitations of our study are the small number of patients and the single-center and retrospective study design. However, the evaluation of mrTRG by a single radiologist and the evaluation of pTRG by a single pathologist, as well as the consistent ChT agent and dosage used were considered strengths of the study in terms of data balance.

\section{Conclusion}

As age increased, pTRG was negatively affected; as the post-nCRT $\mathrm{N}$ stage increased, mrTRG was negatively affected. There was compatibility between mrTRG and pTRG, as expected, but this compatibility was found to be low. A standard should be established for more reliable mrTRG evaluation, and further studies should be performed.

Peer-review: Externally peer-reviewed.

Conflict of Interest: All authors declared no conflict of interest.

Ethics Committee Approval: Institutional Review Board approval was obtained for this study. The study was approved by the Non-Interventional Clinical Research Ethics Committee of Erciyes University Faculty of Medicine (No: 2019/837, Date: $11 / 12 / 2019$ ).

Financial Support: None declared. 
Authorship contributions: Concept - A.A., G.T., İ.P.A.; Design - A.A., S.K.E., İ.P.A.; Supervision - A.A.; Funding - None; Materials - A.A., G.T., E.A.; Data collection and/or processing - A.A., G.T., N.Ö., E.A., S.K.E.; Data analysis and/ or interpretation - A.A., G.T., E.A.; Literature search - A.A., G.T., N.Ö., E.A., S.K.E.; Writing - A.A., N.Ö., İ.P.A.; Critical review - A.A.

\section{References}

1. Horvat N, Petkovska I, Gollub MJ. MR imaging of rectal cancer. Radiol Clin N Am 2018;56:75.

2. Gollub MJ, Arya S, Beets-Tan RG, DePrisco G, Gonen $\mathrm{M}$, Jhaveri $\mathrm{K}$, et al. Use of magnetic resonance imaging in rectal cancer patients: Society of Abdominal Radiology (SAR) rectal cancer disease-focused panel (DFP) recommendations 2017. Abdom Radiol 2018;43(11):2893-902.

3. Horvat N, Rocha CC, Oliveira BC, Petkovska I, Gollub MJ. MRI of rectal cancer: Tumor staging, imaging techniques, and management. Radiographics. 2019;39:367-87.

4. Sauer R, Becker H, Hohenberger W, Rödel C, Wittekind C, Fietkau R, et al. Pre-operative versus postoperative chemoradiotherapy for rectal cancer. $\mathrm{N}$ Engl J Med 2004;351(17):1731-40.

5. Lambregts DM, Boellaard TN, Beets-Tan RG. Response evaluation after neoadjuvant treatment for rectal cancer using modern MR imaging: A pictorial review. Insights Imaging 2019;10(1):15.

6. Braun OM, Neumeister B, Popp W, Scherrer R, Dobrowsky E, Dobrowsky W, et al. Histologic tumor regression grades in squamous cell carcinoma of the head and neck after pre-operative radiochemotherapy. Cancer 1989;63(6):1097-100.

7. Mandard AM, Dalibard F, Mandard JC, Marnay J, Henry-Amar M, Petiot JF, et al. Pathologic assessment of tumor regression after pre-operative chemoradiotherapy of esophageal carcinoma. Clinicopathologic correlations. Cancer 1994;73(11):2680-6.

8. Rödel C, Grabenbauer GG, Kühn R, Papadopoulos T, Dunst J, Meyer M, et al. Combined-modality treatment and selective organ preservation in invasive bladder cancer: Long-term results. J Clin Oncol 2002;20(14):3061-71.

9. Becker K, Mueller JD, Schulmacher C, Ott K, Fink $\mathrm{U}$, Busch R, et al. Histomorphology and grading of regression in gastric carcinoma treated with neoadjuvant chemotherapy. Cancer 2003;98(7):1521-30.

10. Dhadda AS, Dickinson P, Zaitoun AM, Gandhi N, Bessell EM. Prognostic importance of Mandard tumor regression grade following pre-operative chemo/ radiotherapy for locally advanced rectal cancer. Eur J
Cancer 2011;47(8):1138-45.

11. Fokas E, Liersch T, Fietkau R, Hohenberger W, Beissbarth T, Hess C, et al. Tumor regression grading after pre-operative chemoradiotherapy for locally advanced rectal carcinoma revisited: Updated results of the $\mathrm{CAO} /$ ARO/AIO-94 trial. J Clin Oncol 2014;32(15):1554-62.

12. Rodel C, Martus P, Papadoupolos T. Prognostic significant for tumor regression after pre-operative chemoradiotherapy for rectal cancer. J Clin Oncol 2005;23(34):8688-96.

13. Dworak O, Keilholz L, Hoffmann A. Pathological features of rectal cancer after pre-operative radiochemotherapy. Int J Colorectal Dis 1997;12(1):1923.

14. AJCC Cancer Staging Manual. $7^{\text {th }}$ ed. Germany: Springer; 1997. p. 152.

15. Patel UB, Blomqvist LK, Taylor F, George C, Guthrie A, Bees N, et al. MRI after treatment of locally advanced rectal cancer: How to report tumor response-the MERCURY experience. AJR Am J Roentgenol 2012;199(4):486-95.

16. Sclafani F, Brown G, Cunningham D, Wotherspoon A, Mendes LS, Balyasnikova S, et al. Comparison between MRI and pathology in the assessment of tumor regression grade in rectal cancer. $\mathrm{Br} \mathrm{J}$ Cancer 2017;117(10):1478-85.

17. Rengo M, Picchia S, Marzi S, Bellini D, Caruso D, Caterino $\mathrm{M}$, et al. Magnetic resonance tumor regression grade (MR-TRG) to assess pathological complete response following neoadjuvant radiochemotherapy in locally advanced rectal cancer. Oncotarget 2017;8(70):114746-55.

18. Ko HM, Choi YH, Lee JE, Lee KH, Kim JY, Kim JS. Combination assessment of clinical complete response of patients with rectal cancer following chemoradiotherapy with endoscopy and magnetic resonance 1maging. Ann Coloproctol 2019;35(4):202-8.

19. Betge J, Pollheimer MJ, Lindtner RA, Kornprat P, Schlemmer A, Rehak P, et al. Intramural and extramural vascular invasion in colorectal cancer: Prognostic significance and quality of pathology reporting. Cancer 2012;118(3):628-38.

20. Smith NJ, Shihab O, Arnaout A, Swift RI, Brown G. MRI for detection of extramural vascular invasion in rectal cancer. AJR Am J Roentgenol 2008;191:151722.

21. Bugg WG, Andreou AK, Biswas D, Toms AP, Williams SM. The prognostic significance of MRI-detected extramural venous invasion in rectal carcinoma. Clin Radiol 2014;69(6):619-23.

22. Sohn B, Lim JS, Kim H, Myoung S, Choi J, Kim NK, et al. MRI-detected extramural vascular invasion is an independent prognostic factor for synchronous metastasis in patients with rectal cancer. Eur Radiol 
2015;25(5):1347-55.

23. Sun Y, Li J, Shen L, Wang X, Tong T, Gu, Y. Predictive value of MRI-detected extramural vascular invasion in stage T3 rectal cancer patients before neoadjuvant chemoradiation. Diagn Interv Radiol 2018;24(3):12834.

24. Balch GC, De Meo A, Guillem JG. Modern management of rectal canser: A 2006 update. World J Gastroenterol 2006;12(20):3186-95.

25. Taylor FG, Swift RI, Blomqvist L, Brown G. A systematic approach to the interpretation of pre-operative staging MRI for rectal cancer. AJR 2008;191(6):1827-35.

26. Huh JW, Lee JH, Kim HR, Kim YJ. Prognostic significance of lymphovascular or perineural invasion in patients with locally advanced colorectal cancer. Am J
Surg 2013;206(5):758-63.

27. Jhaveri KS, Hosseini-Nik H, Thipphavong S, Assarzadegan N, Menezes RJ, Kennedy ED, et al. MRI detection of extramural venous invasion in rectal cancer: Correlation with histopathology using Elastin Stain. AJR Am J Roentgenol 2016;206(4):747-55.

28. Smith NJ, Barbachano Y, Norman AR, Swift RI, Abulafi AM, Brown G. Prognostic significance of magnetic resonance imaging-detected extramural vascular invasion in rectal cancer. Br J Surg 2008;95(2):229-36.

29. Siddiqui MR, Gormly KL, Bhoday J, Balyansikova S, Battersby NJ, Chand M, et al. Interobserver agreement of radiologists assessing the response of rectal cancers to preoperative chemoradiation using the MRI tumour regression grading (mrTRG). Clin Radiol 2016;71(9):854-62. 